\title{
Circular choosability of graphs
}

\author{
Xuding Zhu \\ Department of Applied Mathematics \\ National Sun Yat-sen University \\ Kaohsiung, Taiwan \\ zhu@math.nsysu.edu.tw
}

\begin{abstract}
This paper discusses the circular version of list coloring of graphs. We give two definitions of the circular list chromatic number (or circular choosability) $\chi_{c, l}(G)$ of a graph $G$ and prove that they are equivalent. Then we prove that for any graph $G, \chi_{c, l}(G) \geq \chi_{l}(G)-1$. Examples are given to show that this bound is sharp in the sense that for any $\epsilon>0$, there is a graph $G$ with $\chi_{c, l}(G)<\chi_{l}(G)-1+\epsilon$. It is also proved that $k$-degenerate graphs $G$ have $\chi_{c, l}(G) \leq 2 k$. This bound is also sharp: for each $\epsilon>0$, there is a $k$-degenerate graph $G$ with $\chi_{c, l}(G) \geq 2 k-\epsilon$. This shows that $\chi_{c, l}(G)$ could be arbitrarily larger than $\chi_{l}(G)$. Finally we prove that if $G$ has maximum degree $k$, then $\chi_{c, l}(G) \leq k+1$.
\end{abstract}

Key words: circular choosability, circular chromatic number, $k$-degenerate graphs

Mathematical Subject Classification: 05C15

\section{Introduction}

Suppose $G=(V, E)$ is a graph and $p \geq 2 q$ are positive integers. A $(p, q)$-coloring of $G$ is a mapping $f: V \rightarrow\{0,1, \cdots, p-1\}$ such that for any edge $u v$ of $G$, $q \leq|f(u)-f(v)| \leq p-q$. Note that a $(p, 1)$-coloring of a graph $G$ is the same as a $p$-coloring of $G$. The circular chromatic number $\chi_{c}(G)$ of $G$ is defined as

$$
\chi_{c}(G)=\inf \{p / q: G \text { admits a }(p, q) \text {-coloring }\} .
$$

It is known [7,8] that for any graph $G, \chi(G)-1<\chi_{c}(G) \leq \chi(G)$. So $\chi_{c}(G)$ is a refinement of $\chi(G)$ and $\chi(G)=\left\lceil\chi_{c}(G)\right\rceil$ is an approximation of $\chi_{c}(G)$.

1 Supported in part by the National Science Council of ROC under grant NSC92-2115-M110-007 
Let $C$ be a set of integers (called colors). A list assignment $L$ is a mapping $L$ which assigns to each vertex $v$ of $G$ a subset $L(v)$ of $C$. The set $L(v)$ is called the set of permissible colors for $v$. An $L$-coloring of $G$ is a mapping $f: V \rightarrow C$ such that for each vertex $v, f(v) \in L(v)$ and for each edge $u v, f(u) \neq f(v)$. The list chromatic number or the choosability $\chi_{l}(G)$ of $G$ is the least integer $k$ such that for any list assignment $L$ for which $|L(v)|=k$ for every vertex $v$ of $G$, there is an $L$-coloring of $G$.

The concept of list coloring has a straightforward circular version.

Definition 1 Suppose $G$ is a graph and $p \geq 2 q$ are positive integers. $A(p, q)$-list assignment $L$ is a mapping which assigns to each vertex $v$ of $G$ a subset $L(v)$ of $\{0,1, \cdots, p-1\}$. An $L$ - $(p, q)$-coloring of $G$ is a $(p, q)$-coloring $f$ of $G$ such that for any vertex $v, f(v) \in L(v)$.

Definition 2 A list-size assignment is a mapping $\ell: V \rightarrow[0, p / q]$. Given a list-size assignment $\ell$, an $\ell$ - $(p, q)$-list assignment is a $(p, q)$-list assignment $L$ such that for each vertex $v,|L(v)| \geq \ell(v) q$. A graph $G$ is called $\ell-(p, q)$-choosable if for any $\ell$ - $(p, q)$-list assignment $L, G$ has has an $L$ - $(p, q)$-coloring.

Circular list coloring was motivated by the needs of inductive proofs in solving circular coloring problems. In an inductive proof, we may need to prove that any $(p, q)$-coloring of a subset $X$ of $V(G)$ can be extended to a $(p, q)$-coloring of $G$. This would be equivalent to prove that for a certain list-size assignment $\ell$, the subgraph $G-X$ is $\ell-(p, q)$-choosable. Such proof techniques are used in [1-4,9]. In [6], the authors gave a characterization of those $\ell$ for which a tree $G$ is $\ell$ $(p, q)$-choosable, and a characterization of those $\ell$ for which an odd cycle $G$ is $\ell-(2 k+1, k)$-choosable.

This paper concerns list-size assignment which are constant mappings. Suppose $t \geq 1$ is a real number and $p \geq 2 q$ are positive integers.

Definition 3 At- $(p, q)$-list assignment is a $(p, q)$-list assignment $L$ such that for every vertex $v,|L(v)| \geq t q$. We say $G$ is circular $t$ - $(p, q)$-choosable if for any $t$ - $(p, q)$ list assignment $L, G$ has an $L-(p, q)$-coloring. We say $G$ is circular $t$-choosable if $G$ is circular $t$ - $(p, q)$-choosable for any positive integers $p \geq 2 q$. The circular list chromatic number (or the circular choosability) of $G$ is defined as

$$
\chi_{c, l}(G)=\inf \{t: G \text { is circular } t \text {-choosable }\} .
$$

Observe that for an integer $k, G$ is $k$-choosable means that $G$ is circular $k$ - $(p, 1)$ choosable for any $p$. Hence $\chi_{l}(G)=\min \{k: G$ is circular $k-(p, 1)$-choosable for any $p\}$.

The circular chromatic number of a graph has a few equivalent definitions [8]. Another frequently used definition is the following: Suppose $r \geq 1$ is a real number. 
Denote by $S(r)$ a circle in the plane with circumference $r$. For two points $x, y$ of $S(r)$, let $|x-y|_{r}$ be the distance on the circle between the two points. We may identify $S(r)$ with the interval $[0, r)$, and imagine that the two end points of the interval are identified. Then $|x-y|_{r}=\min \{|x-y|, r-|x-y|\}$. An $r$-coloring of $G$ is a mapping $f: V \rightarrow S(r)$ such that for any two adjacent vertices $u, v$, $|f(u)-f(v)|_{r} \geq 1$. It is known [8] that for any graph $G, \chi_{c}(G)=\min \{r$ : $G$ has an $r$-coloring\}.

This definition can also be naturally modified to define the circular list chromatic number of a graph. The following version of the circular list chromatic number of a graph is formulated by B. Mohar [5].

Definition 4 A subset $U$ of $S(r)$ is said to be assignable if it is the union of finitely many disjoint open arcs on $S(r)$. The length of an assignable set $U$, denoted by length $(U)$, is the sum of the lengths of the open arcs of $U$. If $G=(V, E)$ is a graph, then a function $L$ that assigns to each vertex $v$ of $G$ an assignable subset $L(v)$ of $S(r)$ is called an circular list assignment (with respect to $r$ ). If for each vertex $v$ of $G, L(v)$ has length at least $t$, then $L$ is called a $t$-circular list assignment (with respect to $r$ ). A circular $L$-coloring of $G$ is a mapping $c$ from $V$ to $S(r)$ such that $c(v) \in L(v)$ for each vertex $v$ of $G$ and for every pair $u, v$ of adjacent vertices of $G,|c(u)-c(v)|_{r} \geq 1$.

We shall prove that for any graph $G$, for any real number $t$, if $G$ is circular $t$ choosable then for $\epsilon>0$ and for any $(t+\epsilon)$-circular list assignment $L, G$ has an circular $L$-coloring. Conversely, if for any $t$-circular list assignment $L, G$ has an circular $L$-coloring, then $G$ is circular $t$-choosable. Therefore the circular list chromatic number of $G$ can be defined as

$\chi_{c, l}(G)=\inf \{t$ : for any $t$-circular list assignment $L, G$ has a circular $L$-coloring $\}$.

Then we study the relation between $\chi_{l}(G)$ and $\chi_{c, l}(G)$. It is proved that for any integer $n$, if $G$ is not $n$-choosable, then $G$ is not circular $n$-choosable. As a consequence, we have $\chi_{c, l}(G) \geq \chi_{l}(G)-1$. On the other hand, it is proved that every $k$ degenerate graph is circular $2 k$-choosable. Moreover, this bound is sharp: for each $\epsilon>0$, there is a $k$-degenerate graph $G$ which is not circular $(2 k-\epsilon)$-choosable. Since every $k$-degenerate graph is $(k+1)$-choosable, it follows that the difference $\chi_{c, l}(G)-\chi_{l}(G)$ can be arbitrarily large. This answers a question of Mohar [5]. Finally, we prove that if $G$ has maximum degree $k$, then $\chi_{c, l}(G) \leq k+1$, which answers another question of Mohar [5]. 


\section{Basic properties}

Let $S(r)$ be a circle of circumference $r$, whose elements of $S(r)$ are identified with points in the interval $[0, r)$. For $a, b \in S(r),(a, b)$ denotes the open interval of $S(r)$ from $a$ to $b$ along the increasing direction. To be precise, if $a \leq b$, then $(a, b)=\{x \in[0, r): a<x<b\}$ (so $(a, a)=\emptyset)$. If $a>b$, then $(a, b)=\{x \in$ $[0, r): a<x<r\} \cup\{x \in[0, r): 0 \leq x<b\}$. The closed interval $[a, b]$ is defined similarly.

First we prove the equivalence of the two definitions of the list circular chromatic of a graph.

Lemma 5 Suppose $G$ is a graph and $t$ is a positive real number. If for every t-circular list assignment $L, G$ has a circular $L$-coloring, then $G$ is circular $t$ choosable. Conversely, if $G$ is circular t-choosable, then for every $\epsilon>0$ for any $(t+\epsilon)$-circular list assignment $L, G$ has a circular L-coloring.

Proof. Assume for every $t$-circular list assignment $L, G$ has a circular $L$-coloring. Let $L^{\prime}$ be an arbitrary $t$ - $(p, q)$-list assignment. Let $L$ be the $t$-circular list assignment with respect to $r=p / q$ defined as $L(v)=\cup_{i \in L^{\prime}(v)}\left(\frac{i}{q}, \frac{i+1}{q}\right)$. By assumption $G$ has a circular $L$-coloring $f$. Let $f^{\prime}(v)=\lfloor f(v) q\rfloor$, then $f^{\prime}$ is an $L^{\prime}-(p, q)$-coloring of $G$.

Conversely, assume that $G$ is a circular $t$-choosable, i.e., for every positive integers $p \geq 2 q$, for every $t$ - $(p, q)$-list assignment $L$ of $G$, there is an $L$ - $(p, q)$-coloring of $G$. Let $\epsilon>0$ and let $L^{\prime}$ be a $(t+\epsilon)$-circular list assignment with respect to $r$. Let $q$ be a fixed positive integer. For a vertex $v$ of $G$, let $L(v)=\{i \in \mathbb{Z}: i / q \in L(v)\}$. Since $L^{\prime}(v)$ has length at least $t+\epsilon$, if $q$ is sufficiently large, then $|L(v)| \geq t q$. Let $p=\lfloor r q\rfloor$, then $L$ is a $t$ - $(p, q)$-list assignment. By assumption, there is an $L$ - $(p, q)$ coloring $f$ of $G$. It is straightforward to verify that $f^{\prime}(v)=f(v) / q$ is a circular $L^{\prime}$-coloring of $G$.

Corollary 6 For any graph $G, \chi_{c, l}(G)=\inf \{t$ : for every $t$-circular list $L, G$ is circular L-colorable $\}$.

In the remainder of this section, we prove some basic properties of circular list chromatic number of a graph. Lemma 7 below follows straightforward from the definition.

Lemma 7 For any graph $G, \chi_{c}(G) \leq \chi_{c, l}(G)$.

Lemma 8 Suppose $G$ is a graph and $k$ is an integer. If $\chi_{l}(G)>k$, then $\chi_{c, l}(G) \geq$ $k$.

Proof. Let $L$ be a list assignment, which assigns to each vertex $v$ of $G$ a set $L(v)$ of at least $k$ permissible colors. Let $r \geq \max \{i+1: i \in L(v)$ for some $v \in V\}$. 
Let $L^{\prime}$ be a circular list assignment with respect to $r$ of $G$ defined as $L^{\prime}(v)=$ $\cup_{i \in L(v)}(i, i+1)$. Then it is easy to see that if $G$ is not $L$-colorable, then $G$ is not circular $L^{\prime}$-colorable.

Corollary 9 For any graph $G, \chi_{c, l}(G) \geq \chi_{l}(G)-1$.

Theorem 10 The odd cycle $C_{2 k+1}$ has $\chi_{c, l}\left(C_{2 k+1}\right)=2+\frac{1}{k}$.

Proof. Since $\chi_{c}\left(C_{2 k+1}\right)=2+\frac{1}{k}$, by Lemma 7, we have $\chi_{c, l}\left(C_{2 k+1}\right) \geq 2+\frac{1}{k}$.

Let $L$ be a $t-(p, q)$-list assignment, where $t \geq \frac{(2 k+1)}{k}$. We shall prove that $C_{2 k+1}$ is $L-(p, q)$-colorable. Assume the vertices of $C_{2 k+1}$ are $v_{0}, v_{1}, \cdots, v_{2 k}$, and the edges are $v_{i} v_{i+1}$ for $i=0,1, \cdots, 2 k$ (addition modulo $2 k+1$ ). Let $c_{0}$ be any color in $L\left(v_{0}\right)$. Let $L^{\prime}\left(v_{1}\right)=L\left(v_{1}\right) \backslash\left[c_{0}-q+1, c_{0}+q-1\right]$, i.e., delete from $L\left(v_{1}\right)$ all the colors that lie in the interval $\left[c_{0}-q+1, c_{0}+q-1\right]$ (calculation modulo $p$ and if $a>b$, then the interval $[a, b]$ denotes the set $\{i: a \leq i \leq p-1$, or $0 \leq i \leq b\}$ ). As $\left[c_{0}-q+1, c_{0}+q-1\right]$ contains $2 q-1$ elements, and $L\left(v_{1}\right)$ contains at least $2 q+\frac{q}{k}$ elements, we conclude that $L^{\prime}\left(v_{1}\right)=L\left(v_{1}\right) \backslash\left[c_{0}-q+1, c_{0}+q-1\right]$ contains at least $1+\left\lceil\frac{q}{k}\right\rceil$ elements. Let $s=\left\lceil\frac{q}{k}\right\rceil$. Assume $\left\{c_{1,1}, c_{1,2}, \cdots, c_{1, s+1}\right\} \subseteq L^{\prime}\left(v_{1}\right)$. Let $L^{\prime}\left(v_{2}\right)=L\left(v_{2}\right) \backslash \cap_{i=1}^{s+1}\left[c_{1, i}-q+1, c_{1, i}+q-1\right]$, i.e., delete from $L\left(v_{2}\right)$ all the colors that lie in the intersection $\cap_{i=1}^{s+1}\left[c_{1, i}-q+1, c_{1, i}+q-1\right]$. We may assume that $c_{1,1}<c_{1,2}<\cdots<c_{1, s+1}$. Then

$$
\cap_{i=1}^{s+1}\left[c_{1, i}-q+1, c_{1, i}+q-1\right] \subseteq\left[c_{1,1}+s+1-q, c_{1,1}+q-1\right] .
$$

This implies that $\cap_{i=1}^{s+1}\left[c_{1, i}-q+1, c_{1, i}+q-1\right]$ contains at most $2 q-s-1$ elements. Hence $L^{\prime}\left(v_{2}\right)$ contains at least $1+2 s$ elements. Recursively, let $L^{\prime}\left(v_{i}\right)=L\left(v_{i}\right) \backslash$ $\cap_{c \in L^{\prime}\left(v_{i-1}\right)}[c-q+1, c+q-1]$. By induction, we can prove that $\left|L^{\prime}\left(v_{i}\right)\right| \geq 1+i s$. In particular, $\left|L^{\prime}\left(v_{2 k}\right)\right| \geq 1+2 k s \geq 1+2 k \frac{q}{k}=1+2 q$. Hence $L^{\prime}\left(v_{2 k}\right) \backslash\left[c_{0}-q+\right.$ $\left.1, c_{0}+q-1\right] \neq \emptyset$. Let $c_{2 k}$ be any color in the set $L^{\prime}\left(v_{2 k}\right) \backslash\left[c_{0}-q+1, c_{0}+q-1\right]$. By definition of $L^{\prime}\left(v_{2 k}\right)$, there is a color $c_{2 k-1} \in L^{\prime}\left(v_{2 k-1}\right)$ such that $c_{2 k} \notin\left[c_{2 k-1}-\right.$ $\left.p+1, c_{2 k-1}+p-1\right]$. Suppose $i \geq 2$ and we have chosen $c_{i} \in L^{\prime}\left(v_{i}\right)$, then chose $c_{i-1}$ to be any color in $L^{\prime}\left(v_{i-1}\right)$ for which $c_{i} \notin\left[c_{i-1}-p+1, c_{i-1}+p-1\right]$. By definition of $L^{\prime}\left(v_{i}\right)$ such a color exists. Then we color $v_{i}$ with color $f\left(v_{i}\right)=c_{i}$ for $i=0,1, \cdots, 2 k$. By the choice of these colors, $f$ is a $(p, q)$ - $L$-coloring $C_{2 k+1}$.

Since $\chi_{l}\left(C_{2 k+1}\right)=3$, Theorem 10 shows that the bound $\chi_{c, l}(G)>\chi_{l}(G)-1$ is sharp.

Suppose $p \geq 2 q$ are positive integers. The graph $K_{p / q}$ has vertices $\{0,1, \cdots, p-1\}$ in which $i \sim j$ if and only if $q \leq|i-j| \leq p-q$. The graphs $K_{p / q}$ are called circular complete graphs. In the study of the circular chromatic number of graphs, they play the same role as that of the complete graphs in the study chromatic number of graphs. Note that odd cycle $C_{2 k+1}$ is isomorphic to $K_{(2 k+1) / k}$. So Theorem 10 determines the circular list chromatic number of some circular complete graphs. It 
would be interesting to determine $\chi_{c, l}\left(K_{p / q}\right)$ for all $p \geq 2 q$.

Question 11 Is it true that $\chi_{c, l}\left(K_{p / q}\right)=p / q$ ?

There are two basic questions concerning the definition of $\chi_{c, l}(G)$ remain open:

Question 12 (1): Is the infimum in the definition always attained, i.e., can it be replaced by the minimum ? (2): Is $\chi_{c, l}$ always a rational number for finite graph $G$ ?

\section{3 circular list coloring of $k$-degenerate graphs}

It is known that for any graph $G, \chi_{c}(G) \leq \chi(G)$ [8]. It is natural to ask, as Mohar did in [5], whether it is true that $\chi_{c, l}(G) \leq \chi_{l}(G)$ for all graphs $G$. This turns out to be false.

Theorem 13 Suppose $G$ is a finite $k$-degenerate graph and $L$ is a $2 k$-circular list assignment of $G$. Then there is a mapping $f$ which assigns to each vertex $v$ of $G$ an open interval $f(v) \subseteq L(v)$ of positive length such that if $v \sim v^{\prime}$, then for any $x \in f(v)$ and $x^{\prime} \in f\left(v^{\prime}\right),\left|x-x^{\prime}\right|_{r} \geq 1$.

Proof. We prove the result by induction on the number of vertices. If $|V(G)|=1$, then this is certainly true. Assume $|V(G)|=n$, and the result holds for any graph $G^{\prime}$ on $n-1$ vertices. Let $v$ be a vertex of $G$ with $d_{G}(v)=k^{\prime} \leq k$. Let $G^{\prime}=G-v$. Then $G^{\prime}$ is $k$-degenerate and hence the required mapping $f$ for $G^{\prime}$ exists by induction hypothesis. Let $v_{1}, v_{2}, \cdots, v_{k^{\prime}}$ be the neighbours of $v$. Assume $f\left(v_{i}\right)=\left(a_{i}, b_{i}\right)$, for $i=1,2, \cdots, k^{\prime}$. Without loss of generality, we may assume that $\left(a_{i}, b_{i}\right)$ has length less than 2 (if $\left(a_{i}, b_{i}\right)$ has length greater than 2 , we can replace it by a sub-interval of $\left(a_{i}, b_{i}\right)$ ). For any point $x \notin\left[b_{i}-1, a_{i}+1\right]$ (here the calculations are modulo $r$ ), there is a point $y \in\left(a_{i}, b_{i}\right)$ such that $|x-y|_{r}>1$. The interval $\left[b_{i}-1, a_{i}+1\right]$ has length less than 2. So the union $\cup_{i=1}^{k^{\prime}}\left[b_{i}-1, a_{i}+1\right]$ has length less than $2 k^{\prime} \leq 2 k$. As $L(v)$ has length at least $2 k$, it follows that there is a point $x \in L(v) \backslash \cup_{i=1}^{k^{\prime}}\left[b_{i}-1, a_{i}+1\right]$. Now for each $i \in\left\{1,2, \cdots, k^{\prime}\right\}$, there is a point $y_{i} \in\left(a_{i}, b_{i}\right)$ such that $\left|x-y_{i}\right|_{r}>1$. Note that the set $L(v) \backslash \cup_{i=1}^{k^{\prime}}\left[b_{i}-1, a_{i}+1\right]$ is open. So for each $i \in\left\{1,2, \cdots, k^{\prime}\right\}$, there is an open interval $\left(c_{i}, d_{i}\right) \subseteq L(v) \backslash \cup_{i=1}^{k^{\prime}}\left[b_{i}-1, a_{i}+1\right]$ containing $x$ and an open interval $\left(a_{i}^{\prime}, b_{i}^{\prime}\right) \subseteq\left(a_{i}, b_{i}\right)$ containing $y_{i}$ such that for any $x^{\prime} \in\left(c_{i}, d_{i}\right)$ and for any $y_{i}^{\prime} \in\left(a_{i}^{\prime}, b_{i}^{\prime}\right),\left|x^{\prime}-y_{i}^{\prime}\right|_{r}>1$. We modify $f$ and then extend it to $V(G)$ by as follows: $f\left(v_{i}\right)=\left(a_{i}^{\prime}, b_{i}^{\prime}\right)$ and $f(v)=\cap_{i=1}^{k^{\prime}}\left(c_{i}, d_{i}\right)$. Then it follows from the definition that the resulting mapping is a required mapping for $G$.

Theorem 14 For any positive integer $k$, for any $\epsilon>0$, there is a $k$-degenerate graph $G$ and $a(2 k-\epsilon)$-circular list assignment $L$ for which there is no circular $L$-coloring of $G$. 
Proof. Let $n$ be an integer such that $n \epsilon>2 k^{2}$. Let $G=K_{k, n^{k}}$ be the complete bipartite graph with partite sets $A=\left\{u_{1}, u_{2}, \cdots, u_{k}\right\}$ and $B=\left\{v_{j_{1}, j_{2}, \cdots, j_{k}}: 1 \leq\right.$ $\left.j_{i} \leq n\right\}$. It is obvious that $G$ is $k$-degenerate. Let $r=2 k(k+1)$. For $i=1,2, \cdots, k$, let $a_{i}=(i-1)(2 k+2)$ and $\delta=\frac{2 k}{n}<\frac{\epsilon}{k}$. Define a circular list assignment $L$ of $G$ as follows: For $i=1,2, \cdots, k$, let $L\left(u_{i}\right)=\left(a_{i}, a_{i}+2 k\right)$. Let $L\left(v_{j_{1}, j_{2}, \cdots, j_{k}}\right)=$ $\cup_{i=1}^{k} A_{i, j_{i}}$, where $A_{i, j_{i}}=\left(a_{i}+j_{i} \delta-1, a_{i}+\left(j_{i}-1\right) \delta+1\right)$. Note that $A_{i, j_{i}}$ is an interval of length $2-\delta$, and that $A_{i, j_{i}} \cap A_{i^{\prime}, j_{i^{\prime}}}=\emptyset$ if $i \neq i^{\prime}$. So $L\left(v_{j_{1}, j_{2}, \cdots, j_{k}}\right)$ has length $(2-\delta) k>2 k-\epsilon$. So $L$ is a $(2 k-\epsilon)$-circular list assignment of $G$. We shall prove that $G$ is not circular $L$-colorable.

Assume to the contrary that $c$ is a circular $L$-coloring of $G$. For $i \in\{1,2, \cdots, k\}$, let $1 \leq j_{i} \leq n$ be an integer such that $c\left(u_{i}\right) \in\left[a_{i}+\left(j_{i}-1\right) \delta, a_{i}+j_{i} \delta\right]$. As $c\left(u_{i}\right) \in L\left(u_{i}\right)=\left(a_{i}, a_{i}+2 k\right)$, such an integer $j_{i}$ exists. For any $i \in\{1,2, \cdots, k\}$, as $v_{j_{1}, j_{2}, \cdots, j_{k}}$ is adjacent to $u_{i}$, we conclude that $c\left(v_{j_{1}, j_{2}, \cdots, j_{k}}\right) \notin\left(a_{i}+j_{i} \delta-1, a_{i}+\left(j_{i}-\right.\right.$ 1) $\delta+1)=A_{i, j_{i}}$ for any $i \in\{1,2, \cdots, k\}$. This is a contradiction, as $L\left(v_{j_{1}, j_{2}, \cdots, j_{k}}\right)=$ $\cup_{i=1}^{k} A_{i, j_{i}}$.

Since $k$-degenerate graphs $G$ have $\chi_{l}(G) \leq k+1$, we conclude that the difference $\chi_{c, l}(G)-\chi_{l}(G)$ can be arbitrarily large. The ratio $\frac{\chi_{c, l}(G)}{\chi_{l}(G)}$ for the graphs given in the proof of Theorem 14 can be arbitrarily close to 2 . It is not clear whether the ratio $\frac{\chi_{c, l}(G)}{\chi_{l}(G)}$ is bounded.

Question 15 Is there a constant $\alpha$ such that for any graph $G, \chi_{c, l}(G) \leq \alpha \chi_{l}(G)$ ? If such a constant exists, what is the smallest $\alpha$ ?

\section{Graphs with maximum degree $k$}

Theorem 16 Suppose $G$ has maximum degree $k$. Then $G$ is circular $(k+1)$ choosable.

Proof. Let $L$ be a $(k+1)$-circular list assignment of $G$, which assigns to each vertex $x$ of $G$ an assignable subset $L(x)$ of a circle $S(r)$ such that length $(L(x)) \geq k+1$. Choose a vertex $v_{0}$. Without loss of generality, we assume that $0 \in L\left(v_{0}\right)$. We color $v_{0}$ with $c\left(v_{0}\right)=0$. We shall color the other vertices one by one. Suppose we have chosen vertices $v_{0}, v_{1}, \cdots, v_{i}$ and colored $v_{j}$ with color $c\left(v_{j}\right)$ for $j=0,1, \cdots, i$. For each vertex $u \notin\left\{v_{0}, v_{1}, \cdots, v_{i}\right\}$, let

$$
L_{i}(u)=\left\{x \in L(u): x-c\left(v_{j}\right) \geq 1, \forall 0 \leq j \leq i, v_{j} \sim u\right\}
$$

and let

$$
\ell_{i}(u)=\inf L_{i}(u)
$$


Let $v_{i+1}$ be a vertex such that

$$
\ell_{i}\left(v_{i+1}\right)=\min \left\{\ell_{i}(u): u \in V(G) \backslash\left\{v_{0}, v_{1}, \cdots, v_{i}\right\}\right\}
$$

Now we color $v_{i+1}$ with color $\ell_{i}\left(v_{i+1}\right)$, i.e., let $c\left(v_{i+1}\right)=\ell_{i}\left(v_{i+1}\right)$. Note that it is possible that $c\left(v_{i+1}\right) \notin L\left(v_{i+1}\right)$, because $L\left(v_{i+1}\right)$ is an open set and $c\left(v_{i+1}\right)$ is the infimum of a subset of $L\left(v_{i+1}\right)$. If $c\left(v_{i+1}\right) \notin L\left(v_{i+1}\right)$, then $c\left(v_{i+1}\right)$ is the left end point of an arc of $L\left(v_{i+1}\right)$. For the moment, we color it with the end point, and we shall modify the coloring at the very end.

We shall prove that at each step, the set $L_{i}(u)$ is non-empty, so the coloring $c$ is well-defined. It follows from the definition that $c\left(v_{0}\right) \leq c\left(v_{1}\right) \leq \cdots \leq c\left(v_{n}\right)$.

Claim 1 Suppose we have colored vertices $v_{0}, v_{1}, \cdots, v_{i}$, and $u$ is an uncolored vertex. Let $L_{i}(u)$ and $\ell_{i}(u)$ be defined as above. If $u$ has t neighbours in $\left\{v_{0}, v_{1}, \cdots, v_{i}\right\}$, then $L(u) \cap\left[0, \ell_{i}(u)\right]$ has length at most $t$, and hence $L_{i}(u)$ has length at least $k+1-t \geq 1$.

Proof. We prove this claim by induction on $i$. First consider the case $i=0$. If $u \not v_{0}$, then $L_{0}(u)=L(u)$ and $\ell_{0}(u)=\inf L(u)$. Hence $L(u) \cap\left[0, \ell_{0}(u)\right]$ has length 0 . If $u \sim v_{0}$, then $L_{0}(u)=\{x \in L(u): x \geq 1\}$ and $\ell_{0}(u)=\inf L_{0}(u)$. Hence $L(u) \cap\left[0, \ell_{0}(u)\right]$ has length at most 1 . Assume $i \geq 1$. Assume that $u$ is an uncolored vertex and has $t$ neighbours in $\left\{v_{0}, v_{1}, \cdots, v_{i}\right\}$.

We assume that $u$ has $t$ neighbours in $\left\{v_{0}, v_{1}, \cdots, v_{i}\right\}$ and has $t^{\prime}$ neighbours in $\left\{v_{0}, v_{1}, \cdots, v_{i-1}\right\}$. By induction hypothesis, $L(u) \cap\left[0, \ell_{i-1}(u)\right]$ has length at most $t^{\prime}$. By the choice of $v_{i}$, we have $\ell_{i-1}(u) \geq \ell_{i-1}\left(v_{i}\right)=c\left(v_{i}\right)$. If $u \neq v_{i}$, then $t=t^{\prime}$, $L_{i}(u)=L_{i-1}(u)$ and $\ell_{i}(u)=\ell_{i-1}(u)$. Hence $L(u) \cap\left[0, \ell_{i}(u)\right]$ has length at most $t^{\prime}=t$. If $u \sim v_{i}$, then $t=t^{\prime}+1$. It follows from the definition that $L(u) \cap$ $\left[c\left(v_{i}\right), \ell_{i}(u)\right]$ has length at most 1 . Since $c\left(v_{i}\right) \leq \ell_{i-1}(u)$ and $L(u) \cap\left[0, \ell_{i-1}(u)\right]$ has length at most $t^{\prime}$, we conclude that $L(u) \cap\left[0, \ell_{i}(u)\right]$ has length at most $t^{\prime}+1=t$. Since $L(u)$ has length at least $k+1$ and $t \leq k$, we conclude that $L_{i}(u)$ has length at least $k+1-t \geq 1$. This completes the proof of Claim 1 .

Thus the coloring scheme produces a coloring $c$ of $G$ such that if $u \sim v$, then $|c(u)-c(v)| \geq 1$, and moreover, $c\left(v_{0}\right) \leq c\left(v_{1}\right) \leq \cdots \leq c\left(v_{n}\right) \leq r-1$ (as $L_{n-1}\left(v_{n}\right)$ has length at least 1$)$. Hence $|c(u)-c(v)|_{r} \geq 1$ for any two adjacent vertices $u$ and $v$. The only problem now is that it may happen that $c(u) \notin L(u)$. As noted above, if $c(u) \notin L(u)$, then $c(u)$ is the left end point of an arc of $L(u)$. If this happens we let $c^{\prime}(u)=c(u)+\delta$ for all $u \in V(G)$. It is easy to see that if $\delta>0$ is small enough, then $c$ is an $L$-coloring of $G$. 


\section{References}

[1] O.V. Borodin, S.J. Kim, A.V. Kostochka, and B.D. West. Homomorphisms from sparse graph with large girth. manuscript, 2003.

[2] G. Fijavž, M. Juvan, B. Mohar, and R. Škrekovski. Circular colorings of planar graphs with prescribed girth. manuscript, 2002.

[3] Anna Galluccio, Luis A. Goddyn, and Pavol Hell. High-girth graphs avoiding a minor are nearly bipartite. J. Combin. Theory Ser. B, 83(1):1-14, 2001.

[4] William Klostermeyer and Cun Quan Zhang. $(2+\epsilon)$-coloring of planar graphs with large odd-girth. J. Graph Theory, 33(2):109-119, 2000.

[5] B. Mohar. Choosability for the circular chromatic number. http://www.fmf.unilj.si/ mohar/Problems/P0201ChoosabilityCircular.html, 2003.

[6] Andre Raspaud and Xuding Zhu. Circular list coloring of trees and cycles. Manuscript, 2003.

[7] A. Vince. Star chromatic number. J. Graph Theory, 12(4):551-559, 1988.

[8] Xuding Zhu. Circular chromatic number: a survey. Discrete Math., 229(1-3):371-410, 2001.

[9] Xuding Zhu. Circular chromatic number of planar graphs of large odd girth. Electron. J. Combin., 8(1):Research Paper 25, 11 pp. (electronic), 2001. 